\title{
ÍNDICE DE TRANSLOCAÇÃO DE NUTRIENTES EM MUDAS DE CEDRO (Cedrela fissilis Vell.) E DE IPÊ-ROXO (Tabebuia impetiginosa (Mart.) Standl.) SUBMETIDAS A DOSES CRESCENTES DE CÁDMIO, NÍQUEL E CHUMBO ${ }^{1}$
}

\author{
Haroldo Nogueira de Paiva ${ }^{2}$, Janice Guedes de Carvalho e José Oswaldo Siqueira ${ }^{3}$
}

\begin{abstract}
RESUMO - Com o objetivo de avaliar os efeitos da toxidez de $\mathrm{Cd}$, $\mathrm{Ni}$ e $\mathrm{Pb}$, mudas de cedro e de ipê-roxo foram cultivadas em solução nutritiva de Clark, em condições de casa de vegetação do Departamento de Ciência do Solo da Universidade Federal de Lavras, com doses crescentes de Cd (0, 22, 44, 88 e $132 \mu \mathrm{mol} / \mathrm{l})$, de Ni $(0,42,84,169$ e $252 \mu \mathrm{mol} / \mathrm{l})$ e de $\mathrm{Pb}(0,48,96,192$ e $288 \mu \mathrm{mol} / \mathrm{l})$, adotando-se para tal um delineamento estatístico de blocos ao acaso, para os três ensaios. Ao final de 60 dias de exposição ao metal pesado, foram feitas avaliações do índice de translocação de macro e de micronutrientes da raiz para a parte aérea, tendo sido constatado que o aumento das doses de $\mathrm{Cd}$, $\mathrm{Ni}$ e $\mathrm{Pb}$ exerceu efeitos sobre os índices de translocação de $\mathrm{P}, \mathrm{K}, \mathrm{Ca}, \mathrm{Mg}, \mathrm{S}, \mathrm{Cu}, \mathrm{Fe}, \mathrm{Mn}$ e $\mathrm{Zn}$, ora positivos, ora negativos, variando com a espécie, o metal pesado e o nutriente.
\end{abstract}

Palavras-chave: Espécies florestais nativas, metal pesado e nutrição de plantas.

\section{TRANSLOCATION INDEX OF NUTRIENTS IN CEDRO (Cedrela fissilis Vell.) AND IPÊ- ROXO (Tabebuia impetiginosa (Mart.) Standl.) SEEDLINGS SUBMITTED TO INCREASING LEVELS OF CADMIUM, NICKEL AND LEAD}

\begin{abstract}
In order to evaluate the effects of $\mathrm{Cd}$, Ni, and Pb toxicity, cedro (Cedrela fissilis Vell.) and ipê-roxo (Tabebuia impetiginosa (Mart.) Standl.) seedlings were cultivated in a Clark nutrient solution under greenhouse conditions at the Soil Science Department of the Federal University of Lavras, and submitted to increasing levels of $\mathrm{Cd}(0,22,44,88$ and $132 \mu \mathrm{mol} / \mathrm{l}), \mathrm{Ni}(0,42,84,169$ and $252 \mu \mathrm{mol} / \mathrm{l})$ and $\mathrm{Pb}(0,48,96,192$ and $288 \mu \mathrm{mol} / \mathrm{l})$, using a statistical design of randomized blocks. At the end of 60 days of exposure to the heavy metals, macro and micronutrient translocation index evaluations were conducted. The results show that, in general, $P, K, C a, M g, S$, $\mathrm{Cu}, \mathrm{Fe}, \mathrm{Mn}$, and $\mathrm{Zn}$ translocation indices are influenced by the increasing levels of $\mathrm{Cd}, \mathrm{Ni}$, and Pb applied. This influence varies according to the species, heavy metal, and nutrient used.
\end{abstract}

Key words: $\quad$ Native forest species, heavy metal and plant nutrition.

\section{INTRODUÇÃO}

A degradação ambiental, promovida principalmente pela ação antrópica, tem exercido forte pressão sobre a qualidade dos ecossistemas. A contaminação do solo e da água por metais pesados tóxicos às plantas e aos animais tem sido uma constante. Neste sentido, a presença de metais como cádmio, chumbo e níquel no solo e na água é, hoje, uma realidade.

As plantas, quando submetidas a ambientes contaminados por metais pesados, apresentam uma série de

1 Recebido para publicação em 20.6.2001.

Aceito para publicação em 20.6.2002.

Parte da tese do primeiro autor, apresentada à Universidade Federal de Lavras.

2 Departamento de Engenharia Florestal da Universidade Federal de Viçosa - UFV, 36571-000 Viçosa-MG, <hnpaiva@mail.ufv.br>. ${ }^{3}$ Departamento de Ciência do Solo da Universidade Federal de Lavras - UFLA, 37200-000 Lavras-MG. 
distúrbios fisiológicos e nutricionais (Mengel \& Kirkby, 1982; Kabata-Pendias e Pendias, 1984; Bergmann, 1992; Marschner, 1995; Soares, 1999; Paiva, 2000). Entre os distúrbios nutricionais tem-se a interferência desses metais sobre a absorção, a translocação e as funções dos nutrientes.

Translocação refere-se ao movimento ou à transferência do íon do local de absorção na raiz para outro ponto qualquer, dentro ou fora da raiz (Faquin, 1994; Malavolta et al., 1997). Índice de translocação refere-se ao movimento ou à transferência do íon da raiz para a parte aérea da planta, sendo representado pela porcentagem da quantidade total absorvida que foi transferida para a parte aérea (Abichequer e Bohnen, 1998).

A presença de metais pesados, ao afetar o índice de translocação, pode provocar uma série de desarranjos fisiológicos nas plantas, pois passa-se a ter deficiência ou excesso de determinados nutrientes na parte aérea, o que prejudica o desenvolvimento e o crescimento normal dessas plantas.

Yang et al. (1996a), trabalhando com plantas de Brassica oleracea (repolho), Trifolium repens (trevobranco), Lolium perenne (azevém) e Zea mays (milho), verificaram que a translocação de $\mathrm{Zn}, \mathrm{Cu}, \mathrm{Fe}$ e $\mathrm{Mn}$ diminuiu com o aumento da concentração de Ni na solução nutritiva, que variou de 0 a $320 \mu \mathrm{mol} / 1$. Grande redução na translocação de $\mathrm{Ca}$ foi verificada para todas as espécies em doses maiores que $120 \mu \mathrm{mol} / \mathrm{l} \mathrm{Ni}$, enquanto para $\mathrm{Mg}$ esta redução foi notada a partir da dose de $30 \mu \mathrm{mol} / 1 \mathrm{Ni}$. Ao contrário, a translocação de $\mathrm{P}$ e de $\mathrm{S}$ em plantas de Lolium perenne e Zea mays aumentou com a dose de $\mathrm{Ni}$ e a translocação de S em Brassica oleracea diminuiu com a crescente dose de Ni. Com a aplicação de $\mathrm{Cd}$, Yang et al. (1996b) constataram que a translocação de $\mathrm{Zn}, \mathrm{Fe}, \mathrm{Cu}, \mathrm{Mn}, \mathrm{Ca}$ e $\mathrm{Mg}$ diminuiu e a translocação de $\mathrm{P}$ aumentou com o incremento das doses de $\mathrm{Cd}$, para plantas de milho, azevém, trevo-branco e repolho, cultivadas em solução nutritiva.

Poucos são os estudos relacionados com a influência da presença de metais pesados sobre o índice de translocação de nutrientes em plantas herbáceas e em gramíneas, espécies amplamente estudadas em ambientes contaminados. Em espécies arbóreas, estes estudos praticamente inexistem, devendo-se empenhar esforços no sentido de conhecer as conseqüências da aplicação e da contaminação por metais pesados, não só em termos de sobrevivência das plantas, mas quanto aos seus efeitos sobre a translocação de nutrientes, fator essencial para o desenvolvimento das espécies vegetais.

R. Árvore, Viçosa-MG, v.26, n. 4, p.467-473, 2002
Como fontes de contaminação do solo por metais pesados podem ser citadas a aplicação de lodo de esgoto, certos fertilizantes fosfatados, corretivos, fungicidas, resíduos industriais e urbanos, além da própria contaminação proveniente da exploração e do beneficiamento de metais (Valadares et al., 1983; Kabata-Pendias e Pendias, 1984; Alloway, 1990; Amaral Sobrinho et al., 1992).

O objetivo do presente trabalho foi verificar a influência da aplicação de cádmio, níquel e chumbo, em solução nutritiva, sobre o índice de translocação de macro e de micronutrientes em mudas de cedro e de ipê-roxo.

\section{MATERIAL E MÉTODOS}

O experimento foi conduzido em casa de vegetação do Departamento de Ciência do Solo da Universidade Federal de Lavras - UFLA, utilizando mudas de cedro (Cedrela fissilis Vell.) e de ipê-roxo (Tabebuia impetiginosa (Mart.) Standl.).

As mudas de cedro e de ipê-roxo foram produzidas em substrato contendo areia lavada, e quando apresentaram altura média de $5 \mathrm{~cm}$ ou dois pares de folhas definitivas foram repicadas para bandejas plásticas com capacidade de 35 1, contendo solução nutritiva de Clark (Clark, 1975), com a concentração de todos os nutrientes reduzida a $20 \%$, com aeração constante, onde permaneceram por 15 dias, quando a solução foi substituída e a concentração de todos os nutrientes foi elevada para $30 \%$ da normal. Trinta dias após a repicagem para as bandejas plásticas, as mudas foram individualizadas em vasos plásticos, com capacidade de $900 \mathrm{ml}$, contendo solução nutritiva a $50 \%$, que foi trocada a cada dez dias. Após 20 dias empregou-se solução normal, e decorridos mais dez dias adicionaram-se os tratamentos, ajustando o pH para 5,5. Para as mudas de ipê-roxo o procedimento foi diferente, pois após 15 dias nas bandejas plásticas com a concentração da solução a $30 \%$ esta foi substituída por outra com concentração de $50 \%$, permanecendo aí por mais 15 dias, quando então foram individualizadas em vasos plásticos, com capacidade de $900 \mathrm{ml}$, contendo solução normal, onde permaneceram por 40 dias, ocasião em que adicionaram-se os tratamentos, ajustando o $\mathrm{pH}$ para 5,5.

Os tratamentos consistiram na aplicação de doses crescentes de cádmio $(0,22,44,88,132 \mu \mathrm{mol} / \mathrm{l})$, usando como fonte o cloreto de cádmio $\left(\mathrm{CdCl}_{2} \cdot 2,5 \mathrm{H}_{2} \mathrm{O}\right)$; de níquel $(0,42,84,169$ e $252 \mu \mathrm{mol} / \mathrm{l})$, usando como fonte 
o nitrato de níquel $\left(\mathrm{NiNO}_{3} \cdot 6 \mathrm{H}_{2} \mathrm{O}\right)$; e de chumbo $(0,48$, 96, 192 e $288 \mu \mathrm{mol} / \mathrm{l})$, usando como fonte o acetato de chumbo $\left(\mathrm{Pb}\left(\mathrm{CH}_{3} \mathrm{COO}\right)_{2} \cdot 3 \mathrm{H}_{2} \mathrm{O}\right)$. Foram conduzidos três ensaios independentes, de forma que não houve combinação entre as doses dos metais pesados. Na preparação de todas as soluções-estoque dos nutrientes e dos metais pesados, empregaram-se reagentes p.a. A solução nutritiva foi preparada com água deionizada, e durante o intervalo de renovação da solução o volume dos vasos foi completado, sempre que necessário, utilizando também água deionizada.

O delineamento estatístico adotado foi o de blocos ao acaso, com cinco tratamentos e cinco repetições, sendo cada repetição representada por um vaso com uma planta, perfazendo, assim, um total de 25 plantas para cada espécie e cada elemento estudado.

As plantas foram mantidas por 60 dias em exposição aos metais pesados, com renovação da solução nutritiva a cada dez dias, mantendo-se o $\mathrm{pH}$ em 5,5 pela adição de $\mathrm{NaOH}$ ou $\mathrm{HCl} 0,1 \mathrm{~mol} / \mathrm{l}$. Após este período procedeu-se à colheita das plantas, separando-as em raiz, caule e folhas.

As raízes, o caule e as folhas foram, então, lavadas em água destilada, postas a secar em estufa com circulação de ar a $65^{\circ} \mathrm{C}$, até peso constante. Determinou-se em balança de precisão $(0,01 \mathrm{~g})$ o peso de matéria seca, que em seguida foi moída em moinho tipo Wiley, equipado com peneira de $0,38 \mathrm{~mm}$, para ser analisada quimicamente.

Após digestão nítrico-perclórica, os teores de $\mathrm{Ca}$, $\mathrm{Mg}, \mathrm{Fe}, \mathrm{Cu}, \mathrm{Mn}$ e $\mathrm{Zn}$ foram determinados por espectrofotometria de absorção atômica. Os teores de $\mathrm{P}$ foram determinados por colorimetria, os teores de $\mathrm{S}$ por turbidimetria e os de K por fotometria de chama (Malavolta et al., 1997).

Os conteúdos de $\mathrm{P}, \mathrm{K}, \mathrm{S}, \mathrm{Ca}, \mathrm{Mg}, \mathrm{Fe}, \mathrm{Cu}, \mathrm{Mn}$ e Zn, na raiz, no caule e nas folhas, foram calculados com base nos teores e nas produções de matéria seca.

De posse dos conteúdos dos vários nutrientes na parte aérea (CPA) e no sistema radicular (CSR), segundo os tratamentos, calcularam-se os índices de translocação (IT, em \%) dos elementos, de acordo com Abichequer e Bohnen (1998), da seguinte forma:

$$
\mathrm{IT}=100 \mathrm{CPA} /(\mathrm{CPA}+\mathrm{CSR})
$$

Os dados foram submetidos à análise de variância e as equações de regressão foram ajustadas por meio do programa estatístico SISVAR.

\section{RESULTADOS E DISCUSSÃO}

$\mathrm{O}$ aumento das doses de $\mathrm{Cd}$, $\mathrm{Ni}$ e $\mathrm{Pb}$ exerceu efeitos significativos sobre os índices de translocação (IT) de P, $\mathrm{K}, \mathrm{Ca}, \mathrm{Mg}, \mathrm{S}, \mathrm{Cu}, \mathrm{Fe}, \mathrm{Mn}$ e $\mathrm{Zn}$, apresentando efeitos diferenciados com a espécie, o metal e o elemento (Quadros 1, 2, 3 e 4). Assim, o Pb reduziu a translocação de $\mathrm{P}$ nas mudas de cedro (Quadros 1 e 2), até a dose de $167 \mu \mathrm{mol} / \mathrm{l} \mathrm{Pb}$, e nas mudas de ipê-roxo (Quadros 3 e 4), até a dose $203 \mu \mathrm{mol} / \mathrm{l} \mathrm{Pb}$, podendo este fato ser explicado, possivelmente, pela formação de fosfato de chumbo na parede celular da raiz, preconizado por Kabata-Pendias e Pendias (1984). O Cd não exerceu efeito significativo, ao passo que a aplicação de Ni provocou redução linear na translocação de $\mathrm{P}$ nas mudas de cedro. Nas mudas de ipê-roxo, o $\mathrm{Cd}$ e o Ni aumentaram a translocação de $\mathrm{P}$. Aumento na translocação de $\mathrm{P}$ foi observado em várias espécies com a aplicação de Cd (Yang et al., 1996b) e de Ni (Yang et al., 1996a), em solução nutritiva.

Nas mudas de cedro, o IT de K e de Ca não foi afetado pela aplicação de $\mathrm{Cd}$ e Ni, enquanto a aplicação de $\mathrm{Pb}$ promoveu aumento na translocação de $\mathrm{K}$ e não apresentou efeito sobre o $\mathrm{Ca}$. O Mg teve sua translocação aumentada linearmente por todos os metais pesados estudados. Quando o comportamento do IT de K, Ca e $\mathrm{Mg}$ nas mudas de ipê-roxo é analisado, verifica-se que o $\mathrm{Pb}$ não exerceu efeito significativo, ao passo que o $\mathrm{Ni} \mathrm{e}$ o Cd o aumentaram. O Cd aumentou o IT de K, Ca e Mg até a dose de 98, 105 e $103 \mu \mathrm{mol} / \mathrm{l} \mathrm{Cd}$, respectivamente, reduzindo-o a partir de então. Estes resultados contrastam-se com outros obtidos em diferentes espécies de plantas, em que a translocação de $\mathrm{Ca}$ e $\mathrm{Mg}$ foi reduzida com a presença de Cd (Yang et al., 1996b) e de Ni (Yang et al., 1996a), mostrando comportamento diferenciando entre as espécies.

A translocação de $\mathrm{S}$ nas mudas de cedro e de ipêroxo aumentou de forma linear com a aplicação de Ni, ao passo que a aplicação de $\mathrm{Pb}$ induziu a uma resposta quadrática, alcançando um mínimo na dose de $116 \mu \mathrm{mol} / \mathrm{l} \mathrm{Pb}$ nas mudas de cedro e $176 \mu \mathrm{mol} / \mathrm{l}$ de $\mathrm{Pb}$ nas mudas de ipê-roxo. Com a aplicação de $\mathrm{Cd}$, as espécies apresentaram diferenças em suas respostas; assim, o IT de $\mathrm{S}$ aumentou linearmente nas mudas de ipê-roxo e apresentou resposta quadrática para o cedro, com um ponto de mínimo na dose de $47 \mu \mathrm{mol} / \mathrm{l} \mathrm{Cd}$. A translocação de S, diante da aplicação de metais pesados, pode ser estimulada ou inibida, dependendo da espécie (Yang et al., 1996a,b).

R. Árvore, Viçosa-MG, v.26, n.4, p.467-473, 2002 
Quadro 1 - Equações de regressão de índice de translocação (IT, em \%) de nutrientes em mudas de cedro, em resposta a diferentes doses de cádmio, níquel e chumbo (em $\mu \mathrm{mol} / \mathrm{l})$

Table 1 - Regression equations of translocation index of nutrients in cedro seedlings, in response to different levels of cadmium, nickel, and lead (in $\mu \mathrm{mol} / \mathrm{l}$ )

\begin{tabular}{|c|c|c|c|}
\hline Nutriente & Metal & Equação de Regressão & $\mathrm{R}^{2}$ \\
\hline $\mathrm{P}$ & $\begin{array}{l}\mathrm{Cd} \\
\mathrm{Ni} \\
\mathrm{Pb}\end{array}$ & $\begin{array}{l}\text { n.s. } \\
\hat{Y}=80,8-0,0334 * * X \\
\hat{Y}=78,4-0,2035^{* *} X+0,000609^{* *} X^{2}\end{array}$ & $\begin{array}{l}0,71 \\
0,76\end{array}$ \\
\hline K & $\begin{array}{l}\mathrm{Cd} \\
\mathrm{Ni} \\
\mathrm{Pb}\end{array}$ & $\begin{array}{l}\text { n.s. } \\
\text { n.s. } \\
\hat{Y}=83,6+0,0233 * * X\end{array}$ & 0,88 \\
\hline $\mathrm{Ca}$ & $\begin{array}{l}\mathrm{Cd} \\
\mathrm{Ni} \\
\mathrm{Pb}\end{array}$ & $\begin{array}{l}\text { n.s. } \\
\text { n.s. } \\
\text { n.s. }\end{array}$ & \\
\hline $\mathrm{Mg}$ & $\begin{array}{l}\mathrm{Cd} \\
\mathrm{Ni} \\
\mathrm{Pb}\end{array}$ & $\begin{array}{l}\hat{Y}=79,4+0,1252 * * X \\
\hat{Y}=74,6+0,0457 * * X \\
\hat{Y}=75,1+0,0544 * * X\end{array}$ & $\begin{array}{l}0,82 \\
0,79 \\
0,88\end{array}$ \\
\hline $\mathrm{S}$ & $\begin{array}{l}\mathrm{Cd} \\
\mathrm{Ni} \\
\mathrm{Pb}\end{array}$ & $\begin{array}{l}\hat{Y}=77,4-0,2492^{* *} \mathrm{X}+0,001634^{* *} \mathrm{X}^{2} \\
\hat{\mathrm{Y}}=70,0+0,0493^{* * X} \\
\hat{\mathrm{Y}}=70,1-0,1198^{* * X} \mathrm{X}+0,000516 \mathrm{X}^{2}\end{array}$ & $\begin{array}{l}0,74 \\
0,96 \\
0,85\end{array}$ \\
\hline $\mathrm{Cu}$ & $\begin{array}{l}\mathrm{Cd} \\
\mathrm{Ni} \\
\mathrm{Pb}\end{array}$ & $\begin{array}{l}\hat{Y}=43,1-5,4421 * * X^{0,5}+0,359941 * * X \\
\hat{Y}=38,2-0,2514 * * X+0,000987 * * X^{2} \\
\text { n.s. }\end{array}$ & $\begin{array}{l}0,95 \\
0,87\end{array}$ \\
\hline $\mathrm{Fe}$ & $\begin{array}{l}\mathrm{Cd} \\
\mathrm{Ni} \\
\mathrm{Pb}\end{array}$ & $\begin{array}{l}\hat{Y}=38,9-0,2392 * * X \\
\hat{Y}=25,1-0,2226 * * X+0,000643 * * X^{2} \\
\text { n.s. }\end{array}$ & $\begin{array}{l}0,93 \\
0,84\end{array}$ \\
\hline $\mathrm{Mn}$ & $\begin{array}{l}\mathrm{Cd} \\
\mathrm{Ni} \\
\mathrm{Pb}\end{array}$ & $\begin{array}{l}\hat{Y}=38,7+0,7335 * * X-0,004358 * * X^{2} \\
\text { n.s. } \\
\hat{Y}=28,7+0,1153 * * X\end{array}$ & $\begin{array}{l}0,81 \\
0,93\end{array}$ \\
\hline $\mathrm{Zn}$ & $\begin{array}{l}\mathrm{Cd} \\
\mathrm{Ni} \\
\mathrm{Pb}\end{array}$ & $\begin{array}{l}\hat{Y}=46,6+0,1836 * * X \\
\hat{Y}=38,9+0,0813 * * X \\
\hat{Y}=34,2+0,1512 * * X\end{array}$ & $\begin{array}{l}0,93 \\
0,92 \\
0,96\end{array}$ \\
\hline
\end{tabular}

** significativo a $1 \%$ de probabilidade, pelo teste $\mathrm{F}$.

n.s. não-significativo a $5 \%$ de probabilidade, pelo teste $\mathrm{F}$.

Quadro 2 - Índice médio de translocação de nutrientes (\%) em mudas de cedro, em resposta a diferentes doses de cádmio, níquel e chumbo $(\mathrm{em} \mu \mathrm{mol} / \mathrm{l})$

Table 2 - Translocation index of nutrients (\%) in cedro seedlings, in response to different levels of cadmium, nickel, and lead (in $\mu \mathrm{mol} / \mathrm{l}$ )

\begin{tabular}{|c|c|c|c|c|c|c|c|c|c|c|}
\hline Metal & Dose & $\mathrm{P}$ & $\mathrm{K}$ & $\mathrm{Ca}$ & $\mathrm{Mg}$ & $\mathrm{S}$ & $\mathrm{Cu}$ & $\mathrm{Fe}$ & $\mathrm{Mn}$ & $\mathrm{Zn}$ \\
\hline \multirow{5}{*}{$\mathrm{Cd}$} & 0 & 84,03 & 89,95 & 88,84 & 76,44 & 79,18 & 43,66 & 43,61 & 33,53 & 44,82 \\
& 22 & 84,46 & 84,14 & 90,90 & 85,98 & 70,38 & 22,94 & 28,93 & 60,74 & 50,38 \\
& 44 & 82,92 & 87,87 & 88,36 & 83,15 & 68,35 & 24,00 & 27,30 & 63,19 & 55,31 \\
& 88 & 85,82 & 87,52 & 89,92 & 93,44 & 70,70 & 25,78 & 18,04 & 63,90 & 67,19 \\
& 132 & 81,48 & 89,92 & 90,61 & 93,92 & 71,96 & 26,67 & 8,44 & 61,81 & 67,65 \\
\hline \multirow{6}{*}{$\mathrm{Ni}$} & 0 & 81,96 & 89,24 & 89,15 & 73,45 & 71,11 & 40,56 & 28,18 & 32,43 & 39,60 \\
& 42 & 76,48 & 87,11 & 87,66 & 76,02 & 70,60 & 26,47 & 12,16 & 38,00 & 42,67 \\
& 84 & 78,96 & 88,98 & 87,44 & 78,75 & 75,25 & 22,67 & 10,82 & 38,42 & 44,91 \\
& 169 & 77,67 & 90,06 & 91,05 & 85,98 & 83,16 & 27,12 & 9,05 & 48,29 & 60,37 \\
& 252 & 71,03 & 87,16 & 87,50 & 83,53 & 77,04 & 36,36 & 18,65 & 42,14 & 51,37 \\
\hline & 0 & 80,64 & 84,19 & 86,76 & 72,03 & 72,40 & 27,84 & 40,19 & 23,75 & 33,04 \\
$\mathrm{~Pb}$ & 48 & 68,66 & 83,73 & 85,78 & 79,11 & 61,66 & 26,76 & 35,07 & 36,15 & 38,30 \\
& 96 & 60,36 & 85,68 & 87,90 & 83,45 & 63,97 & 30,95 & 37,00 & 44,64 & 53,31 \\
& 192 & 66,78 & 89,58 & 87,00 & 84,80 & 68,05 & 22,16 & 38,24 & 51,23 & 66,22 \\
& 288 & 68,63 & 89,67 & 91,01 & 89,96 & 77,69 & 30,05 & 28,77 & 59,74 & 74,97 \\
\hline
\end{tabular}

R. Árvore, Viçosa-MG, v.26, n.4, p.467-473, 2002 
Índice de Translocação de Nutrientes em Mudasd de ...

Quadro 3 - Equações de regressão de índice de translocação (IT, em \%) de nutrientes em mudas de ipê-roxo, em resposta a diferentes doses de cádmio, níquel e chumbo (em $\mu \mathrm{mol} / \mathrm{l})$

Table 3 - Regression equations of translocation index of nutrients in ipê-roxo seedlings, in response to different levels of cadmium, nickel, and lead (in $\mu \mathrm{mol} / \mathrm{l}$ )

\begin{tabular}{|c|c|c|c|}
\hline Nutriente & Metal & Equação de Regressão & $\mathrm{R}^{2}$ \\
\hline $\mathrm{P}$ & $\begin{array}{l}\mathrm{Cd} \\
\mathrm{Ni} \\
\mathrm{Pb}\end{array}$ & $\begin{array}{l}\hat{Y}=60,1+0,4315 * * X-0,002140 * * X^{2} \\
\hat{Y}=61,4+0,0595 * * X \\
\hat{Y}=81,4-0,2769 * * X+0,000682 * * X^{2}\end{array}$ & $\begin{array}{l}0,97 \\
0,86 \\
0,98\end{array}$ \\
\hline K & $\begin{array}{l}\mathrm{Cd} \\
\mathrm{Ni} \\
\mathrm{Pb}\end{array}$ & $\begin{array}{l}\hat{\mathrm{Y}}=81,4+0,3782 * * \mathrm{X}-0,001929 * * \mathrm{X}^{2} \\
\hat{\mathrm{Y}}=89,2+0,0432 * * \mathrm{X} \\
\text { n.s. }\end{array}$ & $\begin{array}{l}0,94 \\
0,82\end{array}$ \\
\hline $\mathrm{Ca}$ & $\begin{array}{l}\mathrm{Cd} \\
\mathrm{Ni} \\
\mathrm{Pb}\end{array}$ & $\begin{array}{l}\hat{Y}=91,1+0,1215 * * X-0,000577 * * X^{2} \\
\hat{Y}=90,1+0,0287 * * X \\
\text { n.s. }\end{array}$ & $\begin{array}{l}0,94 \\
0,91\end{array}$ \\
\hline $\mathrm{Mg}$ & $\begin{array}{l}\mathrm{Cd} \\
\mathrm{Ni} \\
\mathrm{Pb}\end{array}$ & $\begin{array}{l}\hat{Y}=92,5+0,0965 * * X-0,000468 * * X^{2} \\
\hat{Y}=92,7+0,0217 * * X \\
\text { n.s. }\end{array}$ & $\begin{array}{l}0,96 \\
0,91\end{array}$ \\
\hline $\mathrm{S}$ & $\begin{array}{l}\mathrm{Cd} \\
\mathrm{Ni} \\
\mathrm{Pb}\end{array}$ & $\begin{array}{l}\hat{Y}=55,5+0,0829 * * X \\
\hat{Y}=58,7+0,0698 * * X \\
\hat{Y}=75,7-0,2301 * * X+0,000655^{* *} X^{2}\end{array}$ & $\begin{array}{l}0,86 \\
0,83 \\
0,96\end{array}$ \\
\hline $\mathrm{Cu}$ & $\begin{array}{l}\mathrm{Cd} \\
\mathrm{Ni} \\
\mathrm{Pb}\end{array}$ & $\begin{array}{l}\hat{\mathrm{Y}}=\overline{\mathrm{Y}}=35,7 \\
\text { s.a. } \\
\hat{\mathrm{Y}}=51,4-0,0899 * * \mathrm{X}\end{array}$ & 0,77 \\
\hline $\mathrm{Fe}$ & $\begin{array}{l}\mathrm{Cd} \\
\mathrm{Ni} \\
\mathrm{Pb}\end{array}$ & $\begin{array}{l}\hat{\mathrm{Y}}=40,3-0,3908^{* *} \mathrm{X}+0,001757^{* *} \mathrm{X}^{2} \\
\text { n.s. } \\
\hat{\mathrm{Y}}=68,2-0,1202 * * \mathrm{X}\end{array}$ & $\begin{array}{l}0,70 \\
0,81\end{array}$ \\
\hline $\mathrm{Mn}$ & $\begin{array}{l}\mathrm{Cd} \\
\mathrm{Ni} \\
\mathrm{Pb}\end{array}$ & $\begin{array}{l}\text { n.s. } \\
\text { n.s. } \\
\text { n.s. }\end{array}$ & \\
\hline $\mathrm{Zn}$ & $\begin{array}{l}\mathrm{Cd} \\
\mathrm{Ni} \\
\mathrm{Pb}\end{array}$ & $\begin{array}{l}\hat{Y}=70,4+0,3066^{* *} X-0,001552 * * X^{2} \\
\hat{Y}=75,8+0,0548^{* *} X \\
\hat{Y}=89,0-0,1520^{* *} X+0,000460^{* *} X^{2}\end{array}$ & $\begin{array}{l}0,89 \\
0,85 \\
0,96\end{array}$ \\
\hline
\end{tabular}

** significativo a $1 \%$ de probabilidade, pelo teste $\mathrm{F}$.

s.a. sem ajuste.

n.s. não-significativo a $5 \%$ de probabilidade, pelo teste $\mathrm{F}$.

Quadro 4 - Índice médio de translocação de nutrientes (\%) em mudas de ipê-roxo, em resposta a diferentes doses de cádmio, níquel e chumbo (em $\mu \mathrm{mol} / \mathrm{l})$

Table 4 - Translocation index of nutrients (\%) in ipê-roxo seedlings, in response to different levels of cadmium, nickel, and lead (in $\mu \mathrm{mol} / l$ )

\begin{tabular}{|c|c|c|c|c|c|c|c|c|c|c|}
\hline Metal & Dose & $\mathrm{P}$ & $\mathrm{K}$ & $\mathrm{Ca}$ & $\mathrm{Mg}$ & $\mathrm{S}$ & $\mathrm{Cu}$ & $\mathrm{Fe}$ & $\mathrm{Mn}$ & $\mathrm{Zn}$ \\
\hline \multirow{5}{*}{$\mathrm{Cd}$} & 0 & 59,30 & 80,93 & 90,99 & 92,25 & 57,25 & 34,97 & 37,38 & 84,52 & 69,66 \\
& 22 & 69,12 & 88,17 & 93,07 & 94,72 & 54,48 & 44,30 & 41,02 & 87,89 & 75,90 \\
& 44 & 76,63 & 96,97 & 96,32 & 96,33 & 59,62 & 32,50 & 19,38 & 88,36 & 83,75 \\
& 88 & 79,61 & 97,50 & 96,55 & 96,84 & 63,90 & 35,10 & 21,34 & 86,15 & 82,68 \\
& 132 & 80,49 & 98,45 & 97,29 & 97,34 & 66,08 & 31,69 & 19,07 & 85,15 & 84,64 \\
\hline \multirow{5}{*}{$\mathrm{Ni}$} & 0 & 60,60 & 86,72 & 89,44 & 92,22 & 56,59 & 23,63 & 46,92 & 86,78 & 77,01 \\
& 42 & 62,70 & 91,99 & 91,29 & 93,57 & 62,55 & 20,78 & 33,60 & 86,49 & 74,80 \\
& 84 & 67,43 & 94,86 & 93,22 & 95,00 & 69,14 & 26,46 & 40,08 & 85,49 & 81,91 \\
& 16 & 75,09 & 98,00 & 96,17 & 97,31 & 74,58 & 43,59 & 47,51 & 91,84 & 87,34 \\
& 252 & 73,90 & 98,39 & 96,46 & 97,47 & 74,23 & 24,38 & 42,57 & 88,84 & 88,31 \\
\hline \multirow{5}{*}{$\mathrm{Pb}$} & 0 & 80,82 & 97,22 & 96,86 & 97,19 & 74,31 & 53,31 & 74,94 & 93,95 & 88,31 \\
& 48 & 71,64 & 93,63 & 96,85 & 97,33 & 68,50 & 52,77 & 63,71 & 93,03 & 84,46 \\
& 96 & 59,44 & 93,50 & 95,69 & 95,70 & 59,89 & 34,90 & 46,16 & 93,88 & 78,04 \\
& 192 & 53,95 & 93,00 & 95,94 & 95,91 & 54,21 & 30,46 & 43,34 & 93,31 & 76,55 \\
& 28 & 58,22 & 97,31 & 97,11 & 96,81 & 64,50 & 30,98 & 38,20 & 94,66 & 83,67 \\
\hline
\end{tabular}


O cobre teve seu índice de translocação afetado de forma diferenciada com o metal pesado aplicado e a espécie. A presença de $\mathrm{Cd}$ reduziu o IT de $\mathrm{Cu}$ das mudas de cedro até a dose $57,1 \mu \mathrm{mol} / 1 \mathrm{Cd}$, aumentando-o a partir daí, enquanto para as mudas de ipê-roxo não houve efeito significativo. $\mathrm{O} \mathrm{Pb}$ não exerceu efeito significativo nas mudas de cedro, no entanto apresentou resposta linear negativa para as mudas de ipê-roxo. $\mathrm{O} \mathrm{Ni}$ provocou resposta quadrática para o cedro, alcançando um mínimo na dose $127 \mu \mathrm{mol} / \mathrm{l} \mathrm{Ni}$, enquanto não foi encontrado ajuste para os dados de IT de $\mathrm{Cu}$ nas mudas de ipê-roxo. Yang et al. (1996a,b) verificaram que a presença de Cd e de $\mathrm{Ni}$ diminuiu a translocação de $\mathrm{Cu}$, ao passo que Obata e Umebayashi (1997) dizem que a presença de Cd restringe a translocação de $\mathrm{Cu}$ para a parte aérea, o que, no presente caso, não foi necessariamente verdade.

O manganês não apresentou efeito do Ni sobre seu IT, ao passo que com a aplicação de Cd houve resposta quadrática e com a aplicação de $\mathrm{Pb}$ a resposta foi linear positiva nas mudas de cedro. Para as mudas de ipê-roxo, o Mn foi o elemento que sofreu menor interferência em seu IT com a aplicação dos metais; assim, a aplicação de $\mathrm{Cd}, \mathrm{Ni}$ e $\mathrm{Pb}$ não afetou o IT. Em mudas de eucalipto, Soares (1999) detectou pouca influência de Cd e Pb sobre a translocação de Mn, ao passo que Yang et al. (1996a,b) verificaram redução na translocação de $\mathrm{Mn}$, em diferentes espécies, com a aplicação de $\mathrm{Cd}$ e de $\mathrm{Ni}$.

A translocação de Fe foi afetada pelos metais pesados aplicados. O Cd diminuiu o IT de Fe nas duas espécies, tendo este fato também sido observado por Soares (1999), em mudas de eucalipto, e por Yang et al. (1996b), em diferentes espécies. O Ni proporcionou resposta quadrática positiva para as mudas de cedro, com redução no IT de Fe até a dose $173 \mu \mathrm{mol} / 1 \mathrm{Ni}$, enquanto para o ipê-roxo este elemento não afetou a translocação de Fe. A redução na translocação de $\mathrm{Fe}$ foi observada por Yang et al. (1996a) com a aplicação de Ni, em diferentes espécies. Nas mudas de cedro, com a aplicação de $\mathrm{Ni}$, foi observado sintoma de deficiência induzida de $\mathrm{Fe}$, podendo ter sido causado pela redução na translocação deste ou porque a presença de $\mathrm{Ni}$ inibe o metabolismo do Fe (Agarwala et al., 1977). O Pb não afetou o IT de Fe nas mudas de cedro, e fez com que este IT fosse reduzido de forma linear nas mudas de ipê-roxo. Soares (1999) também encontrou redução na translocação de $\mathrm{Fe}$, com a aplicação de $\mathrm{Pb}$ em mudas de eucalipto.

Resposta linear positiva foi observada para o IT de Zn com a aplicação de $\mathrm{Cd}$, Ni e $\mathrm{Pb}$ nas mudas de cedro, enquanto nas mudas de ipê-roxo houve acréscimo no IT de $\mathrm{Zn}$ em resposta à presença de $\mathrm{Cd} \mathrm{e} \mathrm{Ni}$, ao passo que $\mathrm{o}$ $\mathrm{Pb}$ proporcionou resposta quadrática positiva, com redução na translocação de $\mathrm{Zn}$ até a dose $165 \mu \mathrm{mol} / \mathrm{l} \mathrm{Pb}$, aumentando-a a partir de então. Estes resultados mostram que na presença de concentrações elevadas de metais pesados o Zn transloca-se para a parte aérea em maiores proporções que o normal, embora os teores deste metal no caule e na folha tenham sofrido pouca interferência da presença de $\mathrm{Cd}$, $\mathrm{Ni} \mathrm{e} \mathrm{Pb}$. No entanto, estes resultados contrastam com observações de Yang et al. (1996a,b), em que a presença de $\mathrm{Cd}$ e de Ni diminuiu a translocação de Zn.

Os estudos relativos à influência de metais pesados sobre o índice de translocação são restritos, devendo, em pesquisas futuras, este item ser inserido entre seus objetivos.

\section{CONCLUSÃO}

Os resultados obtidos permitem concluir que:

- a aplicação de Cd apresenta respostas diferenciadas, quanto ao índice de translocação de nutrientes, de acordo com a espécie. Neste sentido, aumenta o índice de translocação de $\mathrm{P}, \mathrm{K}, \mathrm{Ca}, \mathrm{Mg}, \mathrm{S}$ e $\mathrm{Zn}$ em mudas de ipêroxo. Para as mudas de cedro, a presença de $\mathrm{Cd}$ não interfere na translocação de $\mathrm{P}, \mathrm{K}, \mathrm{Ca}$ e aumenta a translocação de $\mathrm{Mg}$, Mn e Zn, diminuindo este índice para $\mathrm{S}, \mathrm{Cu}$ e Fe;

- a presença de Ni faz com que o índide de translocação de nutrientes siga a mesma tendência da presença de $\mathrm{Cd}$ para as mudas de ipê-roxo, mas para as mudas de cedro o Ni faz com que haja diminuição no IT de $\mathrm{P}, \mathrm{Cu}$ e Fe e aumento no IT de $\mathrm{Mg}$, S e Zn;

- o chumbo não interfere na translocação de $\mathrm{K}, \mathrm{Ca}, \mathrm{Mg}$ e $\mathrm{Mn}$, mas diminui a translocação de $\mathrm{P}, \mathrm{S}, \mathrm{Cu}, \mathrm{Fe}$ e $\mathrm{Zn}$ em mudas de ipê-roxo. Já para mudas de cedro, o $\mathrm{Pb}$ não interfere no IT de $\mathrm{Ca}, \mathrm{Cu}$ e $\mathrm{Fe}$, no entanto diminui este índice para Pe S, aumentando-o para K, Mg, Mn e Zn; e

- a presença de metais pesados em solução nutritiva faz com que haja resposta diferenciada no índice de translocação dos diferentes nutrientes, de acordo com a espécie.

\section{REFERÊNCIAS BIBLIOGRÁFICAS}

ABICHEQUER, A. D.; BOHNEN, H. Eficiência de absorção, translocação e utilização de fósforo por variedades de trigo. Revista Brasileira de Ciência do Solo, v. 22, n. 1, p. 21-26, 1998. 
AGARWALA, S. C.; BISCHT, S. S.; SHARMA, C. P. Relative effectiveness of certain heavy metals in producing toxicity and symptoms of deficiency in barley. Canadian Journal of Botany, v. 55, n. 10, p. 1299-1307, 1977.

ALlOWAY, B. J. Cadmium. In: ALlOWAY, B. J. (Ed.). Heavy metals in soils. New York: John Willey, 1990. p. $100-121$.

AMARAL SOBRINHO, N. M. B. et al. Heavy metals in fertilizers and liming materials in the state of Minas Gerais. In: ANNUAL CONFERENCE ON TRACE SUBSTANCES IN ENVIRONMENTAL HEALTH, 25., 1991.

Proceeedings... [S.1.:s.n.]: 1992. p. 95-103.

BERGMANN, W. Nutritional disorders of plants: developments, visual and analytical diagnoses. New York: Gustav Fischer Verlang Jena, 1992. 741 p.

CLARK, R. B. Characterization of phosphatase of intact maize roots. Journal of Agriculture and Food Chemistry, v. 23 , n. 3 , p. $458-460,1975$.

FAQUIN, V. Nutrição mineral de plantas. Lavras: ESAL, 1994. 227 p.

KABATA-PENDIAS, A.; PENDIAS, H. Trace elements in soils and plants. Boca Raton: CRC Press, 1984. 315 p.

MALAVOLTA, E.; VITTI, G. C.; OLIVEIRA, S. A. Avaliação do estado nutricional das plantas: princípios e aplicações. 2.ed. Piracicaba: POTAFOS, 1997. 319 p.

MARSCHNER, H. Mineral nutrition of higher plants. 2.ed. London: Academic Press, 1995. 889 p.
MENGEL, K.; KIRKBY, E. A. Principles of plant nutrition. 3.ed. Worblanfen-Bern: International Potash Institute, 1982. 655 p.

OBATA, H.; UMEBAYASHI, M. Effects of cadmium on mineral nutrient concentrations in plants differing tolerance for cadmium. Journal of Plant Nutrition, v. 20, n. 1, p. 97105, 1997.

PAIVA, H. N. Toxidez de Cd, Ni, Pb e Zn em mudas de cedro (Cedrela fissilis Vell.) e ipê-roxo (Tabebuia impetiginosa (Mart.) Standl.). Lavras: Universidade Federal de Lavras, 2000. 283 p. Tese (Doutorado em Fitotecnia) - Universidade Federal de Lavras, 2000.

SOARES, C. R. F. S. Toxidez de zinco, cobre, cádmio e chumbo para o eucalipto em solução nutritiva. Lavras: Universidade Federal de Lavras, 1999. 132 p. Dissertação (Mestrado em Solos e Nutrição de Plantas) - Universidade Federal de Lavras, 1999.

VALADARES, J. M. A. S. et al. Some heavy metals in soils treated with sewage sludge, their effects on yield, and their uptake by plants. Journal of Environmental Quality, v. 12 , n. 1, p. $49-57,1983$.

YANG, X. et al. Plant tolerance to nickel toxicity: II. Nickel effects on influx and transport of mineral nutrients in four plant species. Journal of Plant Nutrition, v. 19, n. 2, p. 265-279, 1996a.

YANG, X. et al. Cadmium effects on influx and tranport of mineral nutrients in plant species. Journal of Plant Nutrition, v. 19, n. 3-4, p. 643-656, 1996b. 